\title{
Search for time correlations between ANTARES neutrino candidates and IceCube/High-Energy Starting Events
}

\section{J. Barrios}

IFIC - Instituto de Física Corpuscular (CSIC - Universitat de València) c/ Catedrático José Beltrán, 2 E-46980 Paterna, Valencia, Spain

E-mail: javier.barrios@ific.uv.es

\section{A. Coleiro}

APC, Univ Paris Diderot, CNRS/IN2P3, CEA/Irfu, Obs de Paris, Sorbonne Paris Cité, France

IFIC - Instituto de Física Corpuscular (CSIC - Universitat de València) c/ Catedrático José Beltrán, 2 E-46980 Paterna,

Valencia, Spain

E-mail: alexis.coleiro@ific.uv.es

\section{G. Illuminati*}

IFIC - Instituto de Física Corpuscular (CSIC - Universitat de València) c/ Catedrático José Beltrán, 2 E-46980 Paterna, Valencia, Spain

E-mail:giulia.illuminatidific.uv.es

\section{on behalf of the ANTARES Collaboration}

\begin{abstract}
We present a search for ANTARES neutrino events in spatial and temporal coincidence with IceCube High-Energy Starting Events (HESE). This analysis uses a maximum likelihood ratio approach based on track events due to muons induced by neutrino interactions observed with ANTARES. Each HESE is treated as the signature of a potential transient neutrino source while the neutrino burst duration, the source direction and the number of ANTARES signal events are obtained as those maximising the likelihood. Before applying the method to the unblinded ANTARES dataset, its sensitivity and discovery potential are computed through dedicated pseudo-experiments. This study provides an effective way to acquire information on the possible origin of the IceCube astrophysical signal from transient sources.
\end{abstract}

35th International Cosmic Ray Conference - ICRC2017

10-20 July, 2017

Bexco, Busan, Korea

${ }^{*}$ Speaker. 


\section{Introduction}

High-energy neutrinos are expected to be produced in cosmic-ray sources. Since they are electrically neutral, weakly interacting and traveling straight from their source without suffering from absorption, neutrinos are unique messengers to further understand the particle acceleration processes in such astrophysical sources.

A high-energy neutrino diffuse flux of cosmic origin has been identified by the IceCube telescope (see e.g. [1]), the sources of which have not been identified yet. In this context, it was pointed out that two of the so-called IceCube High-Energy Starting Events (HESE) occurred within 1 day of each other with a p-value of $1.6 \%$ [2]. Consequently, this was interpreted as the signature of a possible transient point source of very high-energy neutrinos in this part of the sky.

When dealing with transient emissions, the background of atmospheric neutrinos can be significantly reduced using a time-dependent approach that consists in searching for astrophysical neutrinos in smaller time windows around source flares (see e.g. [3]).

Here, we propose a model-independent approach based on the timing properties of both the ANTARES and IceCube data samples. In particular, we search for correlations in space and time between ANTARES muon neutrino candidates and thirteen muon track HESE previously published by the IceCube collaboration. The analysis uses a maximum likelihood ratio approach where each HESE is treated as the signature of a potential transient neutrino source while the neutrino burst duration, the number of ANTARES signal events and the source position are obtained as those maximising the likelihood.

Section 2 reports the IceCube and ANTARES datasets used in this analysis. The method used in the analysis is presented in section 3, while section 4 provides with the preliminary sensitivity and discovery potential, before concluding in section 5 .

\section{Data Sample}

This analysis makes use of the thirteen HESE muon track candidates ${ }^{1}$ detected by the IceCube neutrino telescope within the field-of-view of the ANTARES detector. They have been observed from September 2010 to April 2014 with a median angular error of $\sim 1.3^{\circ}$ (see table 1).

Data of the ANTARES neutrino telescope [5] collected from March 01, 2010 to October 31, 2014 are used in order to overlap the IceCube dataset and to search for flares lasting up to 6 months. Only events reconstructed as muon tracks are included in the dataset. These events are reconstructed by means of a multi-step procedure that estimates the direction and position of the muon using a maximum likelihood fit [6]. Events are selected applying cuts on parameters provided by the reconstruction mechanism: the reconstructed zenith angle $(\cos \theta>-0.1)$, the estimated angular error $\left(\beta<1^{\circ}\right)$ and the parameter that describes the quality of the reconstruction $(\Lambda>-5.2)$. Further cuts are applied on energy-related variables to guarantee the validity of the muon energy estimator employed in this analysis - an approximation of the muon energy deposit per unit path

\footnotetext{
${ }^{1}$ HESE shower candidates observed by IceCube are not taken into account yet due to their larger angular error which requires more computing time for time-dependent analyses.
} 


\begin{tabular}{crrcc} 
HESE ID & $\delta\left[^{\circ}\right]$ & $\alpha\left[^{\circ}\right]$ & $\beta_{\mathrm{IC}}\left[^{\circ}\right]$ & Observation Time (MJD) \\
\hline 3 & -31.2 & 127.9 & 1.4 & 55451.0707482 \\
5 & -0.4 & 110.6 & 1.2 & 55512.5516311 \\
8 & -21.2 & 182.4 & 1.3 & 55608.8201315 \\
13 & 40.3 & 67.9 & 1.2 & 55756.1129844 \\
18 & -24.8 & 345.6 & 1.3 & 55923.5318204 \\
23 & -13.2 & 208.7 & 1.9 & 55949.5693228 \\
28 & -71.5 & 164.8 & 1.3 & 56048.5704209 \\
37 & 20.7 & 167.3 & 1.2 & 56390.1887627 \\
38 & 14.0 & 93.3 & 1.2 & 56470.11038 \\
43 & -22.0 & 206.6 & 1.3 & 56628.56885 \\
44 & 0.0 & -23.3 & 1.2 & 56671.87788 \\
45 & -86.3 & 219.0 & 1.2 & 56679.20447 \\
53 & -37.7 & 239.0 & 1.2 & 56767.06630
\end{tabular}

Table 1: The 13 IceCube HESE muon track candidates $[1,4]$ that are in the field of view of the ANTARES detector. The table gives the equatorial coordinates, the angular error estimate $\beta_{\mathrm{IC}}$ and the date of observation of the event.

length, hereafter referred as $\rho[7,8]$. These cuts were optimized for the latest ANTARES pointsource analysis as detailed in [9]. The distribution of $\Lambda$ and $\beta$ for data, simulated cosmic and atmospheric neutrinos and atmospheric muons is shown in Figure 1. The selection yields a total of 4391 neutrino candidates.

\section{Search Method}

An unbinned method based on an extended maximum likelihood ratio test statistic is employed to look for clusters of events in time and spatial coincidence with the HESE candidates. The used likelihood is defined as

$$
\log \mathcal{L}_{\mathrm{s}+\mathrm{b}}=\sum_{i} \log \left[\mu_{\mathrm{sig}} \mathcal{S}_{i}+\mathcal{N} \mathcal{B}_{i}\right]-\mu_{\mathrm{sig}}
$$

where $\mathcal{S}_{i}$ and $\mathcal{B}_{i}$ are the values of the signal and background probability density functions (PDFs) for the event $i, \mu_{\text {sig }}$ is the number of fitted signal events and $\mathcal{N}$ is the total number of events in the data sample. To improve the signal-to-background discrimination, the combined information of three parameters - direction, energy and observation time - is included in the definition of the signal and background PDFs. For each event $i$, the probability of being reconstructed as signal or background is given by

$$
\begin{aligned}
& \mathcal{S}_{i}=\mathcal{S}^{\text {space }}\left(\Delta \Psi_{i}, \beta_{i}\right) \cdot \mathcal{S}^{\text {energy }}\left(\rho_{i}, \beta_{i} \mid \delta_{i}\right) \cdot \mathcal{S}^{\text {time }}\left(t_{i}\right), \\
& \mathcal{B}_{i}=\mathcal{B}^{\text {space }}\left(\delta_{i}\right) \cdot \mathcal{B}^{\text {energy }}\left(\rho_{i}, \beta_{i} \mid \delta_{i}\right) \cdot \mathcal{B}^{\text {time }}\left(t_{i}\right) .
\end{aligned}
$$



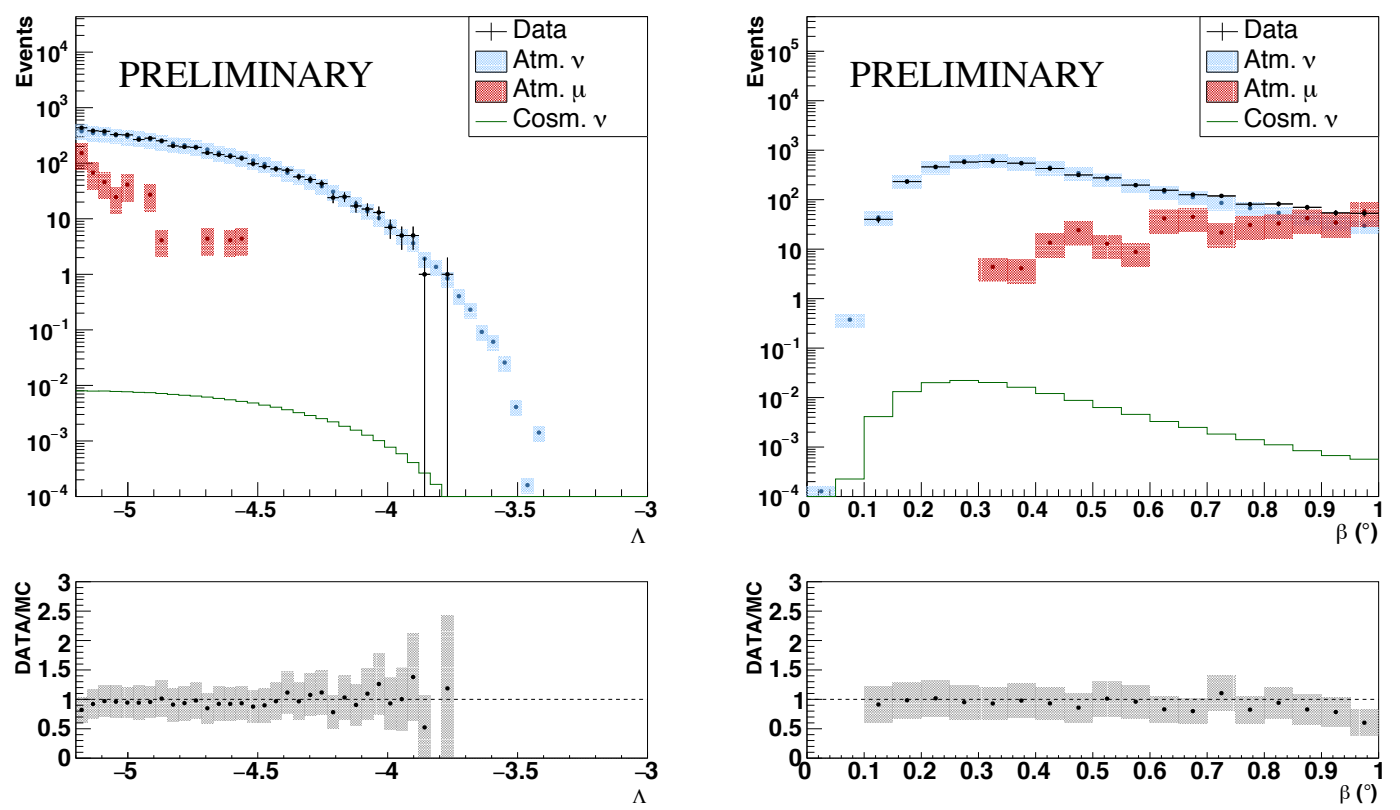

Figure 1: Comparison between data and Monte-Carlo simulation of atmospheric muons, atmospheric neutrinos and expected cosmic signal (assuming a spectrum $d N / d E=10^{-8}(E / \mathrm{GeV})^{-2.5} \mathrm{GeV}^{-1} \mathrm{~cm}^{-2} \mathrm{~s}^{-1}$ ). Distribution of the $\Lambda$ and $\beta$ quality parameters are shown respectively on the left and right. The two bottom plots show the ratio data to Monte-Carlo, where the number of Monte-Carlo events is the sum of atmospheric muons and neutrinos. This figure corresponds to the event distribution after all selection criteria.

respectively. A description of each component is given below.

$\mathcal{S}^{\text {space }}\left(\Delta \Psi_{i}, \beta_{i}\right)$ is a parameterization of the point spread function, i.e. the probability density function of reconstructing the event $i$ at a given angular distance $\Delta \Psi_{i}$ from the true source location. The information of the event angular error estimate $\beta_{i}$ is also included. The shape of this PDF is determined from Monte-Carlo simulations of cosmic neutrinos assuming an $E^{-2.5}$ energy spectrum.

$\mathcal{S}^{\text {energy }}\left(\rho_{i}, \beta_{i} \mid \delta_{i}\right)$ and $\mathcal{B}^{\text {energy }}\left(\rho_{i}, \beta_{i} \mid \delta_{i}\right)$ give the probability for a signal or background event to be reconstructed with an energy estimator $\rho_{i}$ and an angular error estimate $\beta_{i}$. The dependence of the energy estimator on the declination $\delta_{i}$ of the event is taken into account by generating both the PDFs in steps of 0.2 over $\sin \delta$. Monte-Carlo simulations of $E^{-2.5}$ energy spectrum cosmic neutrinos (signal) and of atmospheric neutrinos (background) are used to derive the energy PDFs.

$\mathcal{S}^{\text {time }}\left(t_{i}\right)$ is the signal time-dependent PDF, defined as a Gaussian function : $\mathcal{S}^{\text {time }}\left(t_{i}\right)=\frac{1}{\sqrt{2 \pi} \sigma_{t}} e^{\left(-\frac{\left(t_{i}-t_{H E S E}\right)^{2}}{2 \sigma_{t}^{2}}\right)}$. Here, $t_{i}$ is the arrival time of the event $i, t_{\text {HESE }}$ is the observation time of the HESE candidate, and $\sigma_{t}$ is the fitted flare duration.

$\mathcal{B}^{\text {space }}\left(\delta_{i}\right)$ yields the probability of reconstructing a background event with a certain declination $\delta_{i}$. It is derived from data using the observed declination distribution of the selected events. 
$\mathcal{B}^{\text {time }}\left(t_{i}\right)$ describes the probability to have a background event at a given time $t_{i}$. This PDF is built using the time distribution of both up-going and down-going data events required to satisfy looser selection criteria $\left(\Lambda>-5.4\right.$ and $\left.\beta<1^{\circ}\right)$. This reduces statistical fluctuations while ensuring a good reconstruction quality.

The number of signal events $\mu_{\text {sig }}$ and the flare duration $\sigma_{t}$ are fitted in the likelihood maximization. Moreover, the position in the sky of the fitted source is left free to vary around the position of the IC track within a cone twice as large as the estimated angular error.

The performances of the presented time-dependent analysis are determined by means of pseudoexperiments (PEs), i.e. performing the search for time and spatial correlation on blinded data. In each PE, a fake sky-map containing a number of background events equal to the amount of selected data events and a number of signal events ranging between 0 and 30, is generated. The simulated directions of the background events are randomly drawn from the zenith and azimuth distributions of the selected data. The distribution of the reconstructed zenith angle is shown in Figure 2. Using a time randomly extracted from the time distribution of the data events selected with a looser set of cuts, the equatorial coordinates are computed.

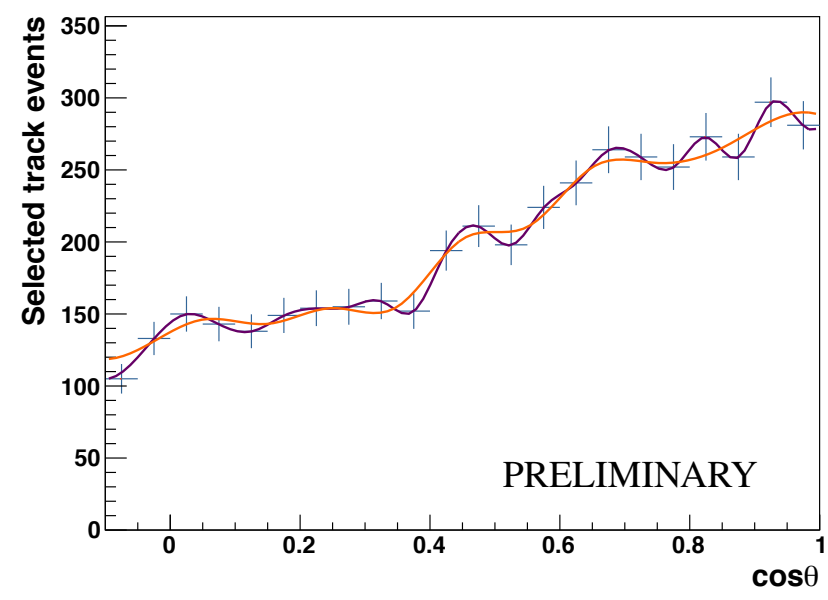

Figure 2: Number of selected data events as a function of the reconstructed zenith angle. The distribution of the background rate is parametrised by two different spline functions, $P(\theta)$ and $O(\theta)$ (the purple and orange lines). In order to account for possible systematic uncertainties on the background, the zenith-dependent distribution of background events, $\mathscr{B}(\theta)$, in each PE is determined as $\mathscr{B}(\theta)=O(\theta)+r \cdot(P(\theta)-O(\theta))$, with $r$ being a random number drawn from a uniform distribution between -1 and 1 .

The simulated signal events are injected around a given source position (one of the 13 HESE candidates) and are assigned a time drawn from a Gaussian function characterized by a mean and a sigma given by the HESE observation time and the tested flare duration respectively. In each PE, the position of the $13 \mathrm{HESE}$ candidates is scanned to look for clustering of events with respect to the expected background. The signal likeness of a cluster is determined by a test statistic computed as

$$
\mathcal{Q}=\log \mathcal{L}_{\mathrm{s}+\mathrm{b}}-\log \mathcal{L}_{\mathrm{b}}
$$


where $\log \mathcal{L}_{\mathrm{b}}$ is the likelihood defined in equation (3.1) evaluated in the background-only case $\left(\mu_{\text {sig }}=0\right)$. Figure 3 shows an example of fake sky-map with the simulated background events and the positions of the 13 HESE.

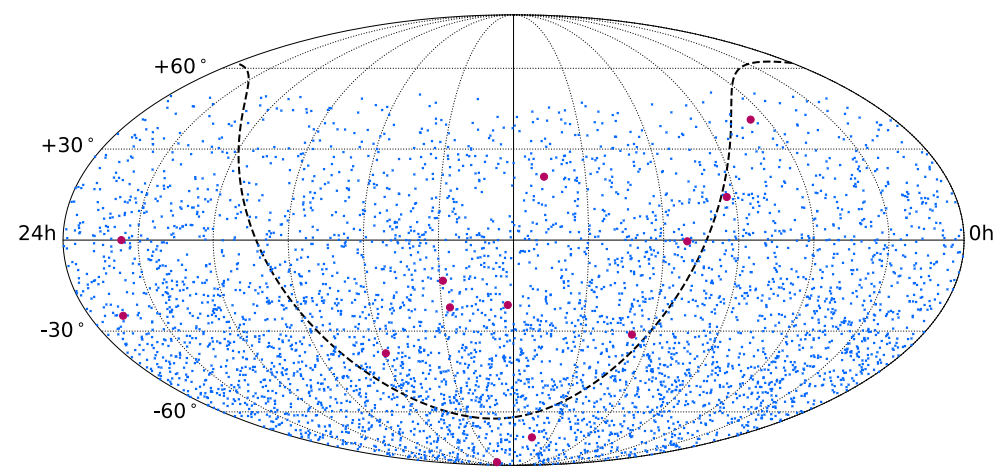

Figure 3: Sky-map of the scrambled background events (blue dots). The position of the 13 HESE tracks is also shown (magenta dots).

\section{Estimated performances}

The detection power (at 50\% confidence level) and sensitivity of the analysis are estimated through pseudo-experiments performed as described above.

Figure 4 shows the preliminary discovery potential and sensitivity for the IceCube HESE with ID 3 as a function of the flare duration.

The ANTARES data are currently blinded. They will be unblinded after having added ANTARES shower events. The 29 high-energy muon events observed by IceCube [10] will be added to the dataset as well before unblinding the analysis.

\section{Conclusions}

A time-correlation analysis between the IceCube/HESE muon track sample and ANTARES muon neutrino candidates observed from 2010 to 2014 has been presented. Using a maximum likelihood ratio approach, it provides with a new way to constrain the origin of the IceCube astrophysical signal from possible transient sources. Limits can be set on the maximal amount of signal events in ANTARES related to a flare of a certain duration. Preliminary results show that sensitivity and discovery potential are better by at least a factor of two compared to time-integrated analyses. In the near future, ANTARES shower events will be included in the analysis and the 29 high-energy muon events observed by IceCube [10] will be added to the dataset as well. The analysis will be unblinded afterwards.

\section{References}

[1] M. G. Aartsen et al. (IceCube collaboration), Observation of High-Energy Astrophysical Neutrinos in Three Years of IceCube Data, Phys.Rev.Lett. 113 (2014) 101101 [arXiv:1405.5303] 


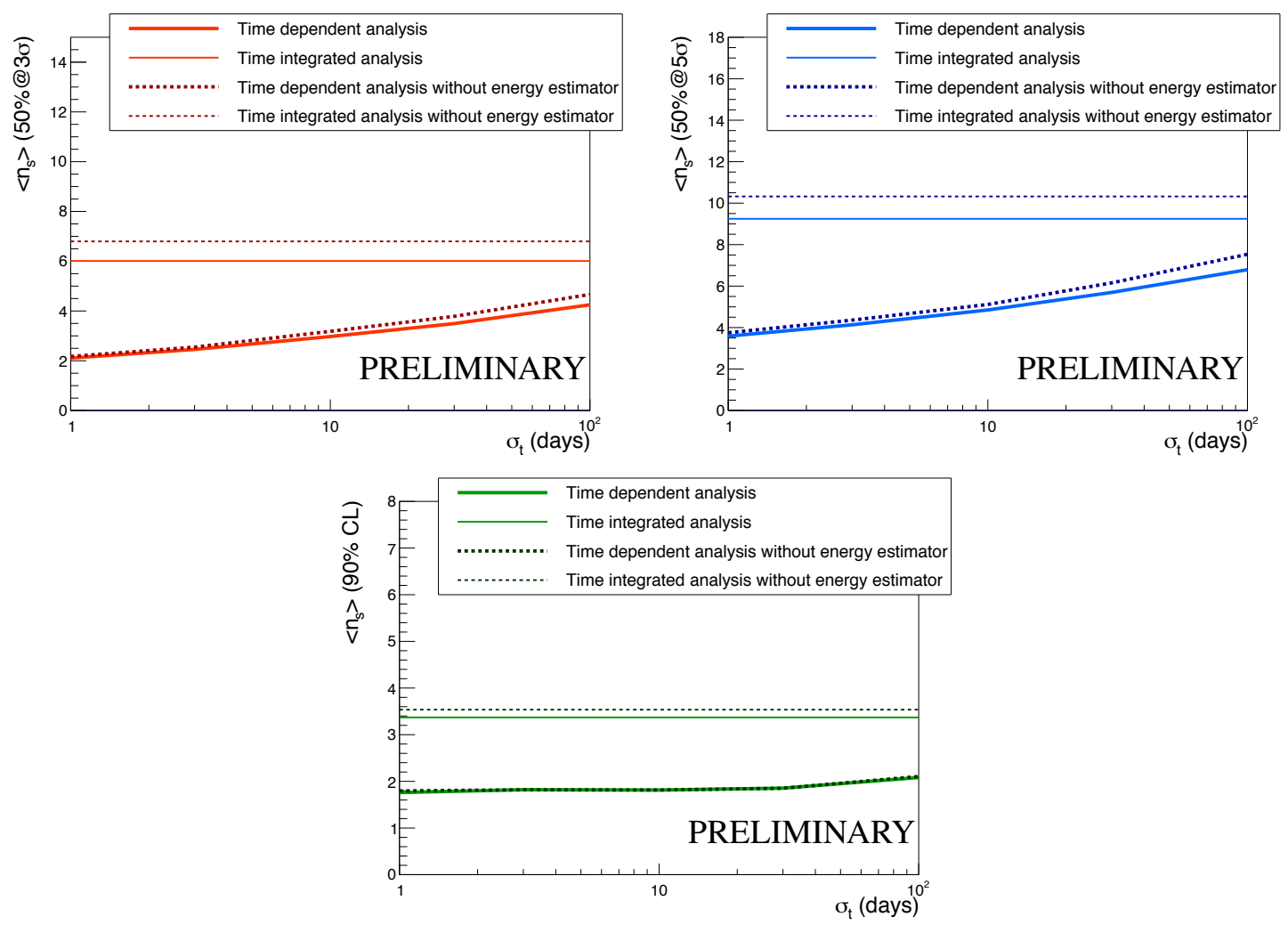

Figure 4: Upper panel: Post-trial $3 \sigma$ and $5 \sigma$ discovery potential (left and right plots respectively) expressed as the mean number of detected events $\left\langle n_{s}\right\rangle$ for IceCube HESE \#3 as a function of the flare duration for the time dependent analysis (solid thick line) and for the time integrated analysis (solid thin line). The curves for the case in which the energy information is not used in the likelihood are also shown. Lower panel: Pretrial sensitivity expressed as the mean number of detected events $<n_{s}>$ for IceCube HESE \#3 as a function of the flare duration for the time dependent analysis (solid thick line) and for the time integrated analysis (solid thin line). The curves for the case in which the energy information is not used in the likelihood are also shown.

[2] Y. Bai et al., Neutrino lighthouse at Sagittarius A* Phys. Rev. D 90 (2014) 063012 [arXiv:1407.2243]

[3] A. Albert et al. (ANTARES collaboration), Time-dependent search for neutrino emission from X-ray binaries with the ANTARES telescope JCAP 4 (2017) 019 [arXiv:1609.07372]

[4] C. Kopper et al., Observation of Astrophysical Neutrinos in Four Years of IceCube Data, in proceedings of 34th International Cosmic Ray Conference (ICRC 2015), POS ICRC2015 (2016) 1081

[5] M. Ageron et al. (ANTARES collaboration), ANTARES: the first undersea neutrino telescope, Nucl.Instrum.Meth. A656 (2011) 11-38 [arXiv:1104.1607]

[6] S. Adrian-Martínez et al. (ANTARES collaboration), Search for Cosmic Neutrino Point Sources with Four Years of Data from the ANTARES Telescope, Astrophys.J. 760 (2012) 53 [arXiv:1207.3105]

[7] F. Schüssler, Energy reconstruction in neutrino telescopes, in proceedings of 33rd International Cosmic Ray Conference (ICRC 2013) 
[8] S. Adrian-Martínez et al. (ANTARES collaboration), Measurement of the atmospheric $v_{\mu}$ energy spectrum from $100 \mathrm{GeV}$ to $200 \mathrm{TeV}$ with the ANTARES telescope, Eur.Phys.J. C73 (2013) 2606 [arXiv:1308.1599]

[9] A. Albert et al. (ANTARES collaboration), First all-flavor Neutrino Point-like Source Search with the ANTARES Neutrino Telescope, [arXiv:1706.01857]

[10] M.G.Aartsen et al. (IceCube collaboration), Observation and Characterization of a Cosmic Muon Neutrino Flux from the Northern Hemisphere using six years of IceCube data, Astrophys. J. 833 (2016) 3 [arXiv:1706.01857] 